\title{
Visual Outcomes of Ultrathin-Descemet Stripping Endothelial Keratoplasty versus Descemet Stripping Endothelial Keratoplasty
}

\author{
Konstantinos Droutsas $\mathbb{D}^{1,},{ }^{1,2}$ Myrsini Petrelli $\mathbb{D}^{1},{ }^{1}$ Dimitrios Miltsakakis, ${ }^{3}$ \\ Konstantinos Andreanos, ${ }_{1}^{1}$ Anastasia Karagianni, ${ }^{3}$ Apostolos Lazaridis $\mathbb{D}^{1},{ }^{1,2}$ \\ Chrysanthi Koutsandrea, ${ }^{1}$ and George Kymionis ${ }^{1,4}$ \\ ${ }^{1}$ First Department of Ophthalmology, National and Kapodistrian University of Athens, General Hospital "G. Gennimatas", \\ Athens, Greece \\ ${ }^{2}$ Department of Ophthalmology, Philipps University, Marburg, Germany \\ ${ }^{3}$ State Ophthalmology Clinic, General Hospital "G. Gennimatas", Athens, Greece \\ ${ }^{4} J u l e s$ Gonin Eye Hospital, Faculty of Biology and Medicine, University of Lausanne, Lausanne, Switzerland \\ Correspondence should be addressed to Konstantinos Droutsas; konstantinos_droutsas@yahoo.gr
}

Received 24 July 2018; Revised 29 September 2018; Accepted 1 October 2018; Published 1 November 2018

Academic Editor: Florence Cabot

Copyright (C) 2018 Konstantinos Droutsas et al. This is an open access article distributed under the Creative Commons Attribution License, which permits unrestricted use, distribution, and reproduction in any medium, provided the original work is properly cited.

\begin{abstract}
Purpose. To examine the impact of graft thickness (GT) on postoperative visual acuity and endothelial cell density after ultrathinDescemet stripping automated endothelial keratoplasty (UT-DSAEK) versus conventional DSAEK. Methods. The medical records of all patients who underwent DSAEK at our institute during a 2-year period were reviewed. After excluding subjects with low visual potential, 34 eyes were divided into two groups based on the postoperative GT as measured with anterior segment optical coherence tomography (AS-OCT): an UT-DSAEK group (GT $\leq 100 \mu \mathrm{m}, n=13$ eyes) and a DSAEK group $(\mathrm{GT}>100 \mu \mathrm{m}, n=21$ eyes). The groups were compared with regard to best-corrected visual acuity (BCVA), subjective refraction, central corneal thickness (CCT), GT, and endothelial cell density (ECD). Results. Preoperative BCVA (logMAR) was $1.035 \pm 0.514$ and $0.772 \pm$ 0.428 for UT-DSAEK and DSAEK, respectively $(P=0.072)$. At 6 months postoperatively, BCVA was $0.088 \pm 0.150$ following UTDSAEK and $0.285 \pm 0.158$ following DSAEK $(P=0.001)$. Conclusion. DSAEK grafts with a thickness under $100 \mu \mathrm{m}$ offered better visual outcomes during the early postoperative period.
\end{abstract}

\section{Introduction}

Over the past decade, Descemet stripping automated endothelial keratoplasty (DSAEK) has surpassed penetrating keratoplasty $(\mathrm{PK})$ as the preferred treatment method for patients with corneal endothelial dysfunction [1]. The numerous advantages of DSAEK over PK include the avoidance of an open sky procedure, absence of suture-related complications, better tectonic and refractive stability, and faster visual rehabilitation [2, 3].

In contrast to PK, where all layers of the host cornea are replaced, DSAEK represents an additive procedure, where a graft consisting of a layer of posterior donor stroma of variable thickness and a layer of healthy corneal endothelium is placed on the posterior surface of the host cornea. This increased corneal thickness may limit the visual outcome after DSAEK [4]. Thus, a trend has emerged [5-7] favouring thinner grafts (i.e., thin and UT-DSAEK) and even alternative surgical procedures, such as Descemet membrane endothelial keratoplasty (DMEK) and pre-Descemet's endothelial keratoplasty (PDEK).

To date, the evidence for differences in visual outcomes depending on GT remains controversial. A number of studies have demonstrated a positive correlation between GT and postoperative visual acuity after DSAEK [8-10], whereas others have not provided supporting data for this hypothesis [11-13]. Thus, the aim of this study was to further elucidate the possible impact of GT on postoperative visual 
acuity by comparing visual outcomes of UT-DSAEK to those of DSAEK.

\section{Materials and Methods}

The medical records of all patients who had undergone DSAEK surgery between October 2015 and August 2017 at a tertiary referral centre (General Hospital "G. Gennimatas," Athens, Greece) were reviewed. Cases with low visual potential (e.g., glaucoma, retinal macular disease, corneal scars, amblyopia) or concurrent ocular surgery (e.g., phacoemulsification) were excluded. Failed or complicated procedures requiring reinterventions such as rebubbling or repeat DSAEK were also excluded. In bilateral cases, only the right was included in the study. All procedures were performed by 3 surgeons (K.D., D.M., and G.K.) with the same surgical technique [14] using precut grafts acquired from an eye bank network that uses a single-pass donor DSAEK graft preparation technique (https://www.sightlife. org/Resources/prepared-corneal-tissue). Notably, the surgeons did not request grafts of a specific thickness range; therefore, the grafts used were of random thickness.

All patients underwent laser iridotomy prior to surgery. The DSAEK procedure was performed under subtenon's block and involved placement of a $4.5 \mathrm{~mm}$ limbal incision (temporally in left eyes and nasally in right eyes), followed by three side ports at 1.00, 6.00, and 9.00. After filling the anterior chamber with air, stripping of the recipient's Descemet membrane was performed using a reverse Sinskey hook. Following removal of the anterior cap of the precut donor tissue, the posterior lamella was mounted on a silicon bank (Geuder AG, Heidelberg, Germany) with the endothelium facing up. The donor lenticule was trephined with an 8.0 to $8.5 \mathrm{~mm}$ punch (Katena, U.S.A.), based on the corneal diameter of the recipient. The graft was then loaded onto a Busin glide (Moria SA, Germany), and a fine intraocular forceps (Moria SA) was used to pull the graft through the limbal incision. Incision wounds were sutured using 100 nylon. Following this, the anterior was filled completely with $100 \%$ air. After 60 to 120 minutes, the patient was examined at the slit lamp and some air was released by gently pressing on a side port in the case of pupillary block.

Preoperative and postoperative best-corrected visual acuity (BCVA), subjective refraction, central corneal thickness (CCT), GT, and endothelial cell density (ECD) were recorded.

CCT and GT were measured using swept-source anterior segment optical coherence tomography (AS-OCT) (DRIOCT Triton, Topcon, Japan). More specifically, the average thickness of 5 points was recorded-measured at the vertex and at $1 \mathrm{~mm}$ located superiorly, inferiorly, temporally, and nasally to the vertex (Figure 1). In addition, four peripheral graft thickness measurements were taken, at two perpendicular axes within $3 \mathrm{~mm}$ from the centre, and the mean value of the latter measurements was calculated $(P)$. Following this, the ratio of central to peripheral graft thickness $(\mathrm{C}: \mathrm{P})$ was calculated. Patients were divided into two groups based on postoperative GT: UT-DSAEK $(\mathrm{GT} \leq 100 \mu \mathrm{m})$ and $\operatorname{DSAEK}(\mathrm{GT}>100 \mu \mathrm{m})$. Donor ECD was extracted from the donor information form, provided by the supplying eye bank. Postoperative ECD was measured with a noncontact specular microscope (EM-3000, Tomey, USA). BCVA was measured in Snellen and converted to a logarithm of the minimum angle of resolution (logMAR) in order to facilitate statistical analysis.

All data were collected with Excel software (version 14, Microsoft Corp.), analyzed with SPSS software (version 17.0, SPSS, Inc.) and reported as central tendency and dispersion. Differences of means between the groups were assessed by the Mann-Whitney $U$ test for independent samples. A $P$ value less than 0.05 was considered statistically significant.

\section{Results}

The study enrolled 43 eyes of 43 patients. Thirteen eyes $(n=13)$ fell within the UT-DSAEK group and 30 eyes $(n=30)$ within the DSAEK group (Table 1$)$. Postoperative GT was $87 \pm 13 \mu \mathrm{m}$ and $145 \pm 21 \mu \mathrm{m}$ following UT-DSAEK and DSAEK, respectively $(P<0.001)$. No significant difference between groups with respect to age and preoperative BCVA was observed ( $P \geq 0.072$, Mann-Whitney $U$ test). The mean corneal donor lenticule C:P ratio in the UT-DSAEK group was $0.65 \pm 0.12(n=12)$ and $0.86 \pm 0.17$ in the DSAEK group $(n=6)(P=0.027)$. In the ultrathin group, mean logMAR improved from 1.035 (range 0.521 to 1.549 ) at baseline to 0.088 (range -0.062 to 0.238 ) at 6 months after surgery. In the DSAEK group, mean logMAR improved from 0.772 (range 0.344 to 1.2 ) at baseline to 0.285 (range -0.127 to 0.443 ) at 6 months. Postoperative $\operatorname{logMAR}$ was significantly better following UT-DSAEK $(P=0.001)$.

A statistically significant moderate positive relationship between postoperative GT and logMAR values $\left(r^{2}=0.423\right.$, $P=0.006$, linear regression analysis) was found in the total group.

Donor ECD was significantly higher in the UT-DSAEK group $(P=0.029)$. Postoperative ECD was $1403 \pm$ 473 cells $/ \mathrm{mm}^{2}$ and $1407 \pm 411$ cells $/ \mathrm{mm}^{2}(P=0.715)$.

\section{Discussion}

DSAEK has replaced PK as the treatment of choice for corneal endothelial dysfunction. Thus, considering the increasing popularity of DSAEK, elucidating the contribution of various factors that influence the final visual outcome is highly significant. A number of factors, including donorrecipient interface, tissue irregularities, anterior corneal scarring, and high-order aberrations, have been suggested as influencing the visual outcome following DSAEK [15-17]. Moreover, a more regular posterior corneal surface has been shown to be achieved with thinner grafts, which, in turn, results in fewer, high-order aberrations. This reduction could explain the faster and better visual recovery observed with thinner DSAEK grafts $[10,18]$.

The aim of this retrospective study was to examine if GT less than $100 \mu \mathrm{m}$ is associated with better postoperative visual acuity, as this could provide guidance for optimizing DSAEK graft thickness. Therefore, we assessed the impact of GT on BCVA following UT-DSAEK versus conventional 


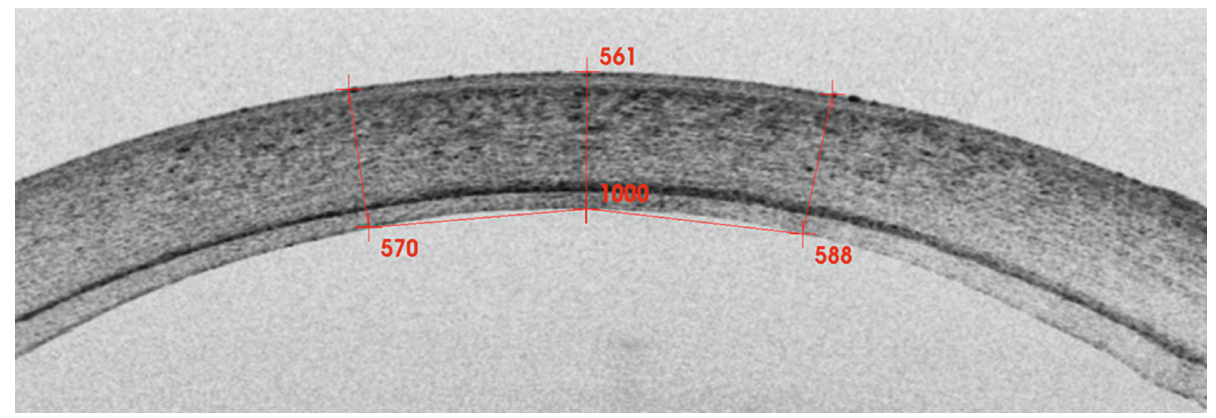

(a)

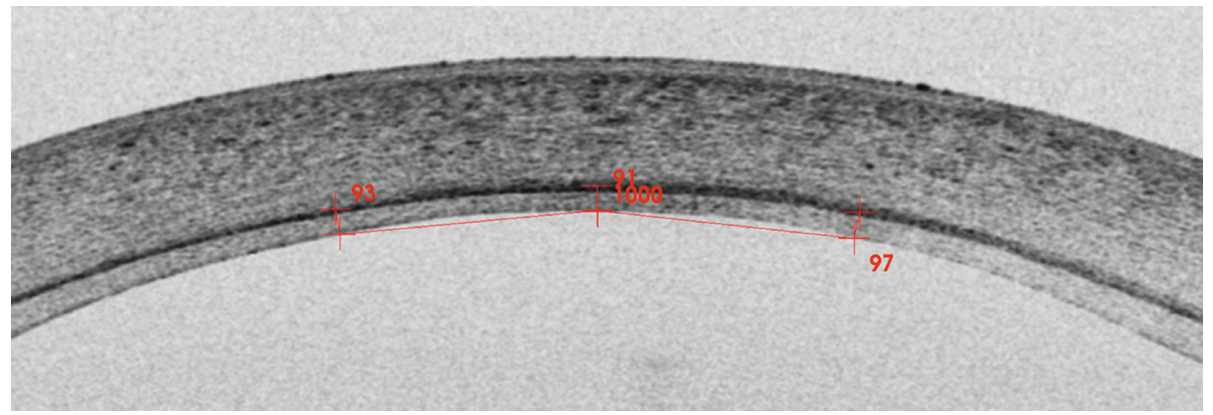

(b)

FIGURE 1: Thickness of a DSAEK graft as measured on an AS-OCT image. Both total (a) and graft (b) corneal thickness were measured at 5 points (at the point of intersection of the measurement's reference axis and the graft and at $1 \mathrm{~mm}$ distance in the superior, inferior, nasal, and temporal meridian). Here are depicted three horizontal points: at the point of intersection of the measurement's reference axis and the graft, and at $1 \mathrm{~mm}$ distance on both sides nasally and temporally to the centre.

TABLE 1: Baseline and 6-month postoperative characteristics.

\begin{tabular}{|c|c|c|c|c|c|}
\hline & \multicolumn{2}{|c|}{ Ultrathin DSAEK $(\mathrm{GT} \leq 100 \mu \mathrm{m})$} & \multicolumn{2}{|c|}{ DSAEK $(\mathrm{GT}>100 \mu \mathrm{m})$} & \multirow{2}{*}{$\begin{array}{c}\text { MWU test } \\
P \text { value }\end{array}$} \\
\hline & Mean \pm SD & $n$ & Mean $\pm \mathrm{SD}$ & $n$ & \\
\hline Age (years) & $72.7 \pm 8.8$ & 13 & $71.4 \pm 7.3$ & 30 & 0.701 \\
\hline Follow-up (months) & $9.3 \pm 4.6$ & 13 & $7.4 \pm 4.4$ & 30 & 0.274 \\
\hline logMARpre & $1.035 \pm 0.514$ & 13 & $0.772 \pm 0.428$ & 30 & 0.072 \\
\hline logMARpost & $0.088 \pm 0.150$ & 13 & $0.285 \pm 0.158$ & 27 & $0.001^{*}$ \\
\hline SEpost (D) & $0.11 \pm 0.54$ & 13 & $0.31 \pm 1.24$ & 26 & 0.227 \\
\hline REFcylpost (D) & $1.173 \pm 0.874$ & 13 & $1.154 \pm 0.863$ & 26 & 0.641 \\
\hline CCTpost $(\mu \mathrm{m})$ & $579 \pm 45$ & 12 & $621 \pm 56$ & 16 & $0.039^{*}$ \\
\hline Gtpost $(\mu \mathrm{m})$ & $87 \pm 13$ & 13 & $145 \pm 21$ & 30 & $0.000^{*}$ \\
\hline ECDpre (cells/mm²) & $2698 \pm 401$ & 13 & $2457 \pm 249$ & 30 & $0.029^{*}$ \\
\hline ECDpost (cells/mm²) & $1403 \pm 473$ & 13 & $1407 \pm 411$ & 19 & 0.715 \\
\hline ECL $(\%)$ & $47 \pm 19$ & 9 & $44 \pm 16$ & 19 & 0.418 \\
\hline
\end{tabular}

*Statistically significant; CCT: central corneal thickness; D: diopters; ECD: endothelial cell density; GT: graft thickness; logMAR: decadic logarithm of the minimal angle of resolution; mo: months; MWU: Mann-Whitney $U$ test; post: postoperatively; pre: preoperatively; REFcyl: refractive cylinder; SE: spherical equivalent.

DSAEK and found significantly better BCVA after UTDSAEK at 6 months after surgery, as well as a moderate positive correlation of GT with BCVA.

Our results are consistent with the findings of previous studies. Neff et al. conducted a retrospective study $(n=33)$ and concluded that grafts that postoperatively were $131 \mu \mathrm{m}$ or thinner had a higher percentage of 20/25 and 20/20 final visual acuity results compared to grafts with a postoperative central GT greater than $131 \mu \mathrm{m}$ [9]. Pogorelov et al. correlated postoperative GT and BCVA 6 months after DSAEK and found a statistically significant relationship $(n=15)$ [8]. Dickman et al. confirmed these results in a larger cohort of eyes without significant comorbidity $(n=79)$ [10]. Acar et al. reported that thinner DSAEK grafts $(\mathrm{GT}<150 \mu \mathrm{m})$ are associated with better visual rehabilitation and less endothelial loss $(n=37)$ [19]. The findings of a recent randomized multicenter clinical trial indicated that UT-DSAEK, compared to DSAEK, promotes faster and better visual acuity results with a similar endothelial cell loss at 1 year postoperatively $(n=66)$ [20]. Finally, results from a relatively 
recent review of the literature and meta-analysis suggest a weak relationship between GT and BCVA following DSAEK [21].

Nevertheless, several other studies have not provided evidence supporting a clear association between GT and visual acuity following DSAEK [11-13, 22-24].

To our knowledge, there is currently no consensus on the basis upon which the categorization of GT should be done. The exact thickness defining UT, as opposed to conventional DSAEK, is not uniform in the literature and has been described variously as sub $-130 \mu \mathrm{m}$ or sub- $100 \mu \mathrm{m}[25,26]$. Moreover, some studies refer to the GT measurement immediately after graft preparation [13, 23, 27], while others use the measurement after surgery in vivo $[10,22,24]$. Here, we have used the definition of UT-DSAEK based on postoperative GT measurement as previously reported by others [4].

In order to assess donor corneal lenticule morphology, we calculated the C:P ratio of the DSAEK graft, an index that represents the ratio of the central graft thickness to the peripheral as formerly described [28]. Interestingly, the C:P ratio was found to be higher in the DSAEK group.

Different endothelial keratoplasty techniques have not shown significantly different ECL up to date. The slightly higher but not significant ECL in the UT-DSAEK group (47\% versus $44 \%$ following UT-DSAEK and DSAEK, respectively) agrees with previous observations [20].

Limitations of this study include its retrospective nature and the small number of the studied eyes. Randomized, prospective studies with larger sample size are required in order to examine the relationship between both preoperative and postoperative donor thickness and postoperative vision and to confirm the theory that better visual outcomes can be achieved with the use of ultrathin DSAEK grafts.

\section{Data Availability}

The data used to support the findings of this study are available from the corresponding author upon request.

\section{Conflicts of Interest}

The authors declare that there are no conflicts of interest regarding the publication of this article.

\section{References}

[1] M. O. Price and F. W. Price Jr., "Endothelial keratoplasty-a review," Clinical \& Experimental Ophthalmology, vol. 38, no. 2, pp. 128-140, 2010.

[2] M. A. Terry, N. Shamie, E. S. Chen, K. L. Hoar, and D. J. Friend, "Endothelial keratoplasty a simplified technique to minimize graft dislocation, iatrogenic graft failure, and pupillary block," Ophthalmology, vol. 115, no. 7, pp. 11791186, 2008.

[3] E. S. Chen, M. A. Terry, N. Shamie, K. L. Hoar, and D. J. Friend, "Descemet-stripping automated endothelial keratoplasty," Cornea, vol. 27, no. 5, pp. 514-520, 2008.

[4] A. M. J. Turnbull, M. Tsatsos, P. N. Hossain, and D. F. Anderson, "Determinants of visual quality after endothelial keratoplasty," Survey of Ophthalmology, vol. 61, no. 3, pp. 257-271, 2016.

[5] M. Busin, S. Madi, P. Santorum, V. Scorcia, and J. Beltz, "Ultrathin descemet's stripping automated endothelial keratoplasty with the microkeratome double-pass technique," Ophthalmology, vol. 120, no. 6, pp. 1186-1194, 2013.

[6] G. R. J. Melles, T. S. Ong, B. Ververs, and J. van der Wees, "Descemet membrane endothelial keratoplasty (DMEK)," Cornea, vol. 25, no. 8, pp. 987-990, 2006.

[7] A. Agarwal, H. S. Dua, P. Narang et al., "Pre-Descemet's Endothelial Keratoplasty (PDEK)," British Journal of Ophthalmology, vol. 98, no. 9, pp. 1181-1185, 2014.

[8] P. Pogorelov, C. Cursiefen, B. O. Bachmann, and F. E. Kruse, "Changes in donor corneal lenticule thickness after Descemet's Stripping Automated Endothelial Keratoplasty (DSAEK) with organ-cultured corneas," British Journal of Ophthalmology, vol. 93, no. 6, pp. 825-829, 2009.

[9] K. D. Neff, J. M. Biber, and E. J. Holland, "Comparison of central corneal graft thickness to visual acuity outcomes in endothelial keratoplasty," Cornea, vol. 30, no. 4, pp. 388-391, 2011.

[10] M. M. Dickman, Y. Y. Y. Cheng, T. T. J. M. Berendschot, F. J. H. M. van den Biggelaar, and R. M. M. A. Nuijts, "Effects of graft thickness and asymmetry on visual gain and aberrations after Descemet Stripping Automated Endothelial Keratoplasty," JAMA Ophthalmology, vol. 131, no. 6, p. 737, 2013.

[11] M. O. Price and F. W. Price, "Descemet's stripping with endothelial keratoplasty," Ophthalmology, vol. 113, no. 11, pp. 1936-1942, 2006.

[12] K. A. Ahmed, J. W. McLaren, K. H. Baratz, L. J. Maguire, K. M. Kittleson, and S. V. Patel, "Host and graft thickness after Descemet stripping endothelial keratoplasty for fuchs endothelial dystrophy," American Journal of Ophthalmology, vol. 150, no. 4, pp. 490-497.e2, 2010.

[13] H. Van Cleynenbreugel, L. Remeijer, and T. Hillenaar, "Descemet stripping automated endothelial keratoplasty: effect of intraoperative lenticule thickness on visual outcome and endothelial cell density," Cornea, vol. 30, no. 11, pp. 1195-1200, 2011.

[14] M. Busin, P. R. Bhatt, and V. Scorcia, "A modified technique for Descemet membrane stripping automated endothelial keratoplasty to minimize endothelial cell loss," Archives of Ophthalmology, vol. 126, no. 8, p. 1133, 2008.

[15] S. V. Patel, K. H. Baratz, D. O. Hodge, L. J. Maguire, and J. W. McLaren, "The effect of corneal light scatter on vision after Descemet stripping with endothelial keratoplasty," Archives of Ophthalmology, vol. 127, no. 2, p. 153, 2009.

[16] M. Rudolph, K. Laaser, B. O. Bachmann, C. Cursiefen, D. Epstein, and F. E. Kruse, "Corneal higher-order aberrations after descemet's membrane endothelial keratoplasty," Ophthalmology, vol. 119, no. 3, pp. 528-535, 2012.

[17] M. Dirisamer, J. Parker, M. Naveiras et al., "Identifying causes for poor visual outcome after DSEK/DSAEK following secondary DMEK in the same eye," Acta Ophthalmologica, vol. 91, no. 2, pp. 131-139, 2013.

[18] M. Busin and E. Albé, "Does thickness matter," Current Opinion in Ophthalmology, vol. 25, no. 4, pp. 312-318, 2014.

[19] B. T. Acar, M. O. Akdemir, and S. Acar, "Visual acuity and endothelial cell density with respect to the graft thickness in Descemet's stripping automated endothelial keratoplasty: one year results," International Journal of Ophthalmology Press, vol. 7, no. 6, pp. 974-979, 2014. 
[20] M. M. Dickman, P. J. Kruit, L. Remeijer et al., “A randomized multicenter clinical trial of ultrathin Descemet Stripping Automated Endothelial Keratoplasty (DSAEK) versus DSAEK," Ophthalmology, vol. 123, no. 11, pp. 2276-2284, 2016.

[21] K. Wacker, W. M. Bourne, and S. V. Patel, "Effect of graft thickness on visual acuity after Descemet stripping endothelial keratoplasty: a systematic review and meta-analysis," American Journal of Ophthalmology, vol. 163, pp. 18-28, 2016.

[22] A. J. Shinton, M. Tsatsos, A. Konstantopoulos et al., "Impact of graft thickness on visual acuity after Descemet's stripping endothelial keratoplasty," British Journal of Ophthalmology, vol. 96, no. 2, pp. 246-249, 2012 Feb.

[23] M. A. Terry, M. D. Straiko, J. M. Goshe, J. Y. Li, and D. DavisBoozer, "Descemet's stripping automated endothelial keratoplasty: the tenuous relationship between donor thickness and postoperative vision," Ophthalmology, vol. 119, no. 10, pp. 1988-1996, 2012.

[24] M. A. Woodward, D. Raoof-Daneshvar, S. Mian, and R. M. Shtein, "Relationship of visual acuity and lamellar thickness in Descemet stripping automated endothelial keratoplasty," Cornea, vol. 32, no. 5, pp. e69-e73, 2013.

[25] M. Busin, A. K. Patel, V. Scorcia, and D. Ponzin, "Microkeratome-assisted preparation of ultrathin grafts for Descemet stripping automated endothelial keratoplasty," Investigative Opthalmology \& Visual Science, vol. 53, no. 1, p. 521, 2012.

[26] M. Hsu, W. L. Hereth, and M. Moshirfar, "Double-pass microkeratome technique for ultra-thin graft preparation in Descemet's stripping automated endothelial keratoplasty," Clinical Ophthalmology Dove Press, vol. 6, pp. 425-432, 2012.

[27] P. M. Phillips, L. J. Phillips, and C. M. Maloney, "Preoperative graft thickness measurements do not influence final BSCVA or speed of vision recovery after Descemet stripping automated endothelial keratoplasty," Cornea, vol. 32, no. 11, pp. 1423-1427, 2013.

[28] S. H. Yoo, G. D. Kymionis, A. A. Deobhakta et al., "One-year results and anterior segment optical coherence tomography findings of Descemet stripping automated endothelial keratoplasty combined with phacoemulsification," Archives of Ophthalmology American Medical Association, vol. 126, no. 8, p. 1052, 2008. 


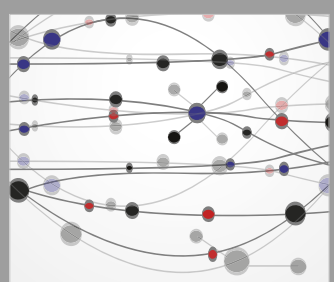

The Scientific World Journal
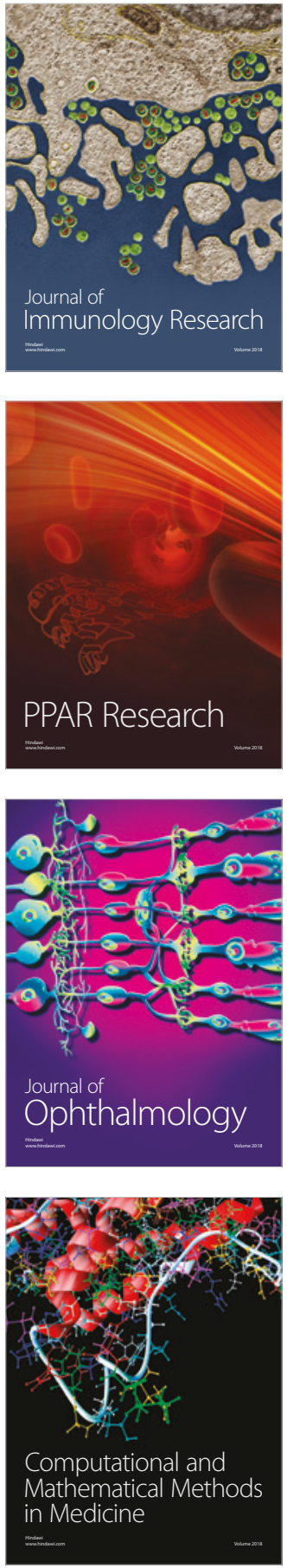

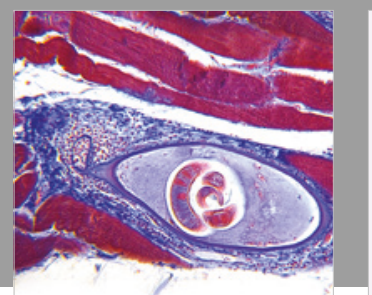

Gastroenterology Research and Practice

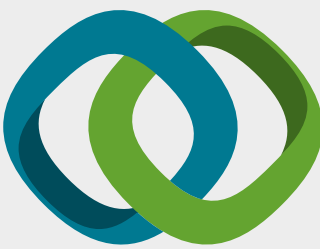

\section{Hindawi}

Submit your manuscripts at

www.hindawi.com
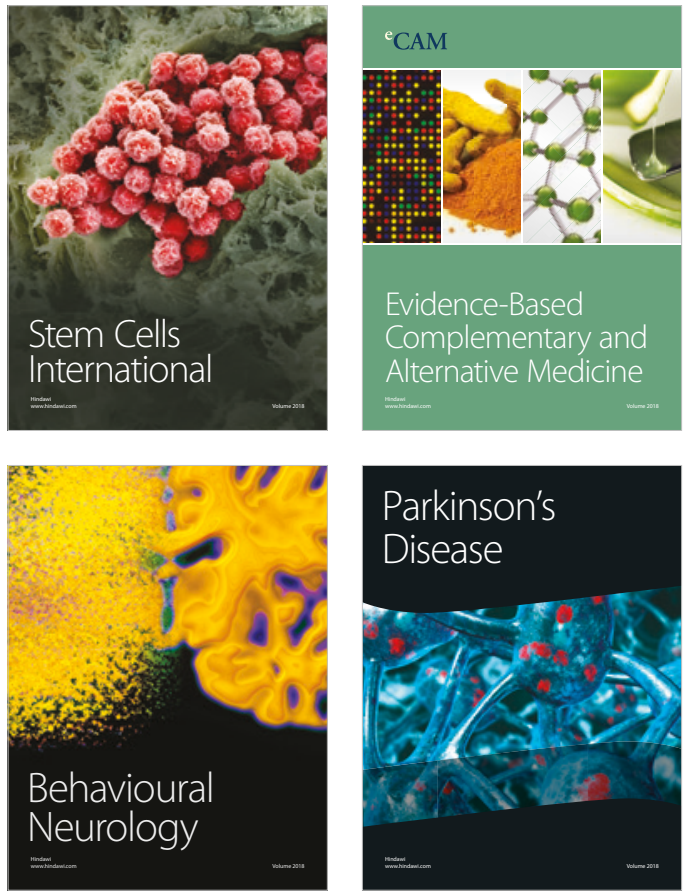

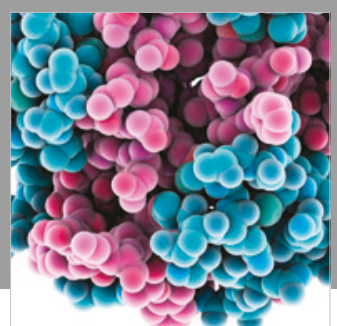

ournal of

Diabetes Research

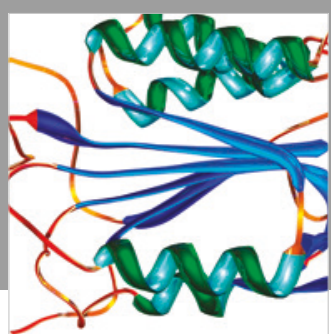

Disease Markers
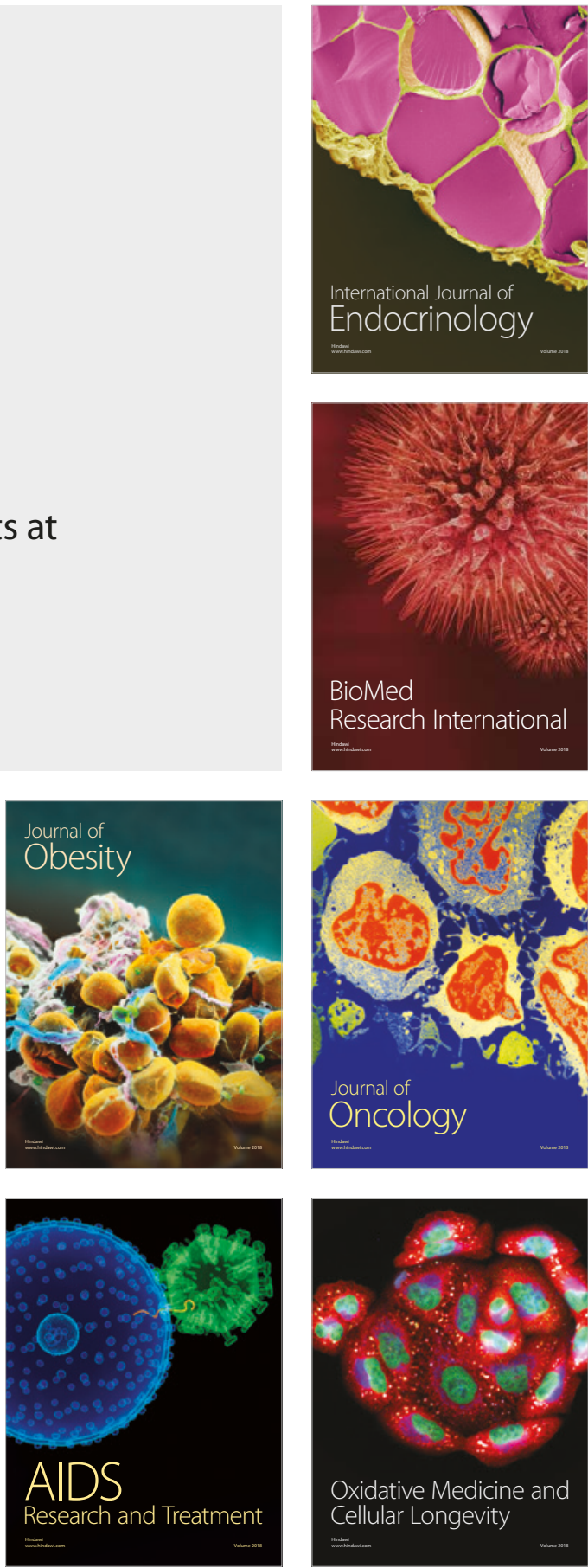